BOOK REVIEWS

\title{
Hereditary hearing loss - the updated resource book more needed than ever
}

\section{• Hereditary hearing loss and its syndromes}

Edited by H Toriello, W Reardon and RJ Gorlin. Oxford University Press, Oxford Monographs on Medical Genetics No. 50, 2nd Edition, 2004

..........

Lisbeth Tranebjærg

European Journal of Human Genetics (2005) 13, 889-890. doi:10.1038/sj.ejhg.5201396

$\mathrm{S}$ ince the first edition of this excellent reference book came in 1995, the new information gained from international research in genetics of hearing and hearing loss have increased tremendously. William Reardon is a new member of the three-man editorial staff, with the other two being involved also in the first edition. The outline and style of the book and the clinical angle as main focus have been kept unchanged.

The book has increased from 460 to 500 pages. The organization of the different types of hearing impairment has remained constructed around different organ system as in the first edition and in the very first renowned book by Bruce Königsmark in 1976. Minor reorganization of the chapters, and the invitation of several leading scientist to author individual chapters keep the book at the cutting edge of this rapidly growing field. The most remarkable editorial change is the addition of Chapter 5, reviewing, in a comprehensive manner, nonsyndromic hearing loss, with a strong focus on clinical features.

Chapters 1-4 cover history, principles of syndrome diagnosis, epidemiological, and etiological aspects of hearing impairment and embryology of the ear. It would have been extremely helpful if recommendations as elaborated by American College of Medical Genetics for etiological evaluation had been commented in Chapter 2. Chapter 4 is an expert's instructive presentation of the embryology of the ear, but more focus on the identification of genes of developmental significance would have added a deeper molecular understanding of these basic processes. Chapter 5 is a valuable addition to the previous edition, containing overview with several comprehensive tables combining the gene localization data with clinical information about the audiological pattern of hearing impairment. The pace of the identification of such loci and genes is illustrated by the fact that one table lists DFNA1-41, and the web-based primary source for this information (http://www.uia.ac.be/dnalab/hhh/ as of January 1, 2005) includes 54 DFNA entities. Similarly, there are now 53 DFNB types representing autosomal recessive hearing impairment, with only 33 entities published at the closing of the editorial work of this book. By virtue of the rapid pace of the advancements of the identification of new loci and genes, an electronic version would be welcomed.

The extensive coverage of DFNB1 elaborating on connexin 26-related hearing impairment is appropriate considering the frequency of GJB2 mutations worldwide, but connexin-related hearing impairment might even have justified a section of its own including more elaborated genotype-phenotype aspects. Chapter 6 is a very extensive and clinically extremely well-documented chapter on hearing impairment associated with external ear abnormalities.

Chapter 7 deals with those types of hearing deficiencies combined with eye disorders, which is a field in particularly rapid advancement because of the description of an increasing numbers of genetic causes of deaf-blindness. Chapter 8 , covering 100 pages, presents an extremely valuable collection of the large number of musculoskeletal disorders with hearing impairment attached. Chapter 9 describes hearing impairment disorders with renal affection in a very innovative manner with extensive coverage of the genetic aspects, probably reflecting the in-depth knowledge of the authors.

A broader description of the present molecular understanding of the shared developmental pathways between kidney and inner ear would have been welcomed.

Chapter 10 covers conditions with neurological abnormalities, growing in numbers along with a deeper neuropathological understanding of many of the disorders. The dilemma of where to list specific conditions representing multiorgan disorders has been solved in a balanced way throughout the new edition. Chapter 11 is a new creation focusing on cardiac defects, and largely stemming from the chapter of miscellaneous syndromes no longer included.

Chapter 12 on endocrine disorders includes two very well-known syndromes, Wolfram syndrome and Pendred syndrome, which have been extensively genetically investigated since 1st edition. The large number of patients with either WFS1 or SLC26A4 mutations reported and the extremely variable clinical presentation would have justified an even broader description of the spectrum of mutations versus clinical implications in order to guide clinicians to select the most likely patients for molecular investigations in clinical audiology.

Chapters 13, 14, and 15 deal with metabolic disorders, skin abnormalities, and chromosomal disorders in association with hearing impairment. Despite their briefness, they all give important lists of important conditions to consider. It is worth noticing however, that Williams syndrome, recently recognized to include associated audiological affection, is completely missing. This still reflects that 
accumulated long-term audiological investigation of several syndromes is insufficiently described, and therefore not available. It should be acknowledged that space is allocated to mention the value of Mendelian cytogenetics as a tool to identify new genes by identifying the disruption of genes located in the breakpoints of balanced inherited translocations, cosegregating with a specific clinical condition.

In general, the degree of update in terms of proven genetic heterogeneity and functional studies of identified gene products varies between the different chapters, reflecting the different backgrounds of the specific authors. Only some chapters consistently use MIM (Mendelian Inheritance in Man) numbers, which are very helpful for a smooth comparison between different types of electronic and printed sources.

The appearance of an appendix at the end of each chapter listing other conditions with similar clinical affection but described in another chapter helps in leading the readers to relevant places, when searching for a diagnosis, based on clinical findings.

Many chapters contain surprisingly few references to web-based sources, which would have been much appreciated. A chapter or section on its own could even be considered.

In an increasing number of hearing impairment syndromes, there are now data on temporal bone histopathology and the outcome of treatment by cochlear implant, which would have been worthwhile to quote, since the book has a clinical approach.

\section{Genetic epidemiology}

$\bullet \bullet \bullet \bullet \bullet \bullet \bullet \bullet \bullet \bullet \bullet \bullet \bullet \bullet \bullet \bullet \bullet \bullet \bullet \bullet \bullet \bullet \bullet \bullet \bullet \bullet \bullet \bullet \bullet \bullet \bullet \bullet \bullet \bullet \bullet \bullet \bullet \bullet$

Human genome epidemiology

Edited by MJ Khoury, J Little and W Burke. Oxford University Press Inc., New York, 2004

...............

Claude Stoll

European Journal of Human Genetics (2005) 13, 890

doi:10.1038/sj.ejhg.5201397

$\mathrm{D}$ iscoveries occurring in the field of human genetics are expected to play a central role in medicine and public health by providing genetic information for disease prediction and prevention. The translation of genetic advances into actions to improve health and prevent diseases is depending on scientific information from multiples disciplines. Epidemiology plays a central role in this effort.

In this book, the authors aim to show how the epidemiologic approach will play an important role in the continuum from gene discovery to the development and applications of genetic test.
The authors call this continuum human genome epidemiology (HuGE) to denote an evolving field of inquiry that uses systematic applications of epidemiologic methods to assess the impact of human genetic variation on health and disease.

HuGE is a unique text in which the leaders from a diverse group of disciplines have joined their efforts to create the first comprehensive text on HuGE. This 549page text is divided into four sections, which are further subdivided into 29 chapters.

Part I describes genomic technologies and their applications, and summarizes
In general, Hereditary Hearing Loss and its Syndromes is still a Bible for all those professionals dealing with hearing impairment, and this new, revised edition is warmly recommended. The pace and amount at which the scientific data is accumulating almost makes this task unsurmountable, and definitely calls for some electronic solution in the future. Despite minor editorial discrepancies between different chapters, the book is still a 'must' on the bookshelf for both clinically and scientifically oriented professionals in this field

Lisbeth Tranebjoerg is at the Department of Audiology, Bispebjerg Hospital, Copenhagen NV, Denmark. E-mail: tranebjaerg@imbg.ku.dk

the ethical, legal, and social issues for conducting epidemiologic studies of the human genome. Part II addresses epidemiologic approaches to the studies of genotypes in populations and their relation to diseases. Part III deals with the application of epidemiologic methods to assess genetic information for clinical and public health applications. Part IV uses case studies to illustrate concepts discussed in the first three sections in relation to specific disease examples including gene-environment interactions (pesticides and oral contraceptive use), chronic diseases (colon cancer, Alzheimer disease, cardiovascular disease, breast cancer, and hemochromatosis), occupational exposures, newborn screening issues (fragile $\mathrm{X}$ syndrome and hearing loss), and infectious disease (HIV-1 infection).

HuGE is an invaluable resource for a wide audience including epidemiologists, clinical geneticists, molecular geneticists, cytogeneticists, statistical geneticists, population geneticists, services geneticists, medical students, residents, and basic and clinical scientists

Claude Stoll is at the Genetique Medicale, Faculte de Medecine, Strasbourg, France. E-mail: Claude.Stoll@medecine.u-strasbg.fr 